Conductivity of N-Dimensional Composites Containing Hyperspherical Inclusion Author(s): A. S. Sangani

Reviewed work(s):

Source: SIAM Journal on Applied Mathematics, Vol. 50, No. 1 (Feb., 1990), pp. 64-73

Published by: Society for Industrial and Applied Mathematics

Stable URL: http://www.jstor.org/stable/2102100

Accessed: 05/03/2012 13:28

Your use of the JSTOR archive indicates your acceptance of the Terms \& Conditions of Use, available at http://www.jstor.org/page/info/about/policies/terms.jsp

JSTOR is a not-for-profit service that helps scholars, researchers, and students discover, use, and build upon a wide range of content in a trusted digital archive. We use information technology and tools to increase productivity and facilitate new forms of scholarship. For more information about JSTOR, please contact support@jstor.org. 


\title{
CONDUCTIVITY OF N-DIMENSIONAL COMPOSITES CONTAINING HYPERSPHERICAL INCLUSION*
}

\author{
A. S. SANGANI $\dagger$
}

\begin{abstract}
A problem of determining the macroscopic or effective thermal conductivity of an $\mathrm{N}$ dimensional composite medium containing $\mathrm{N}$-dimensional nonoverlapping hyperspherical inclusions is considered. Since the macroscopic conductivity is expected to become less sensitive to the detailed spatial distribution of the inclusions for $N \geqq 4$, only the special case of periodic arrangement of the inclusions is considered. An expression for the macroscopic conductivity correct to $O\left(\chi^{3 N+8}\right), \chi$ being the ratio of "diameter" of the inclusions to the spacing between them, is derived and the numerical results for the conductivity are presented as a function of $\chi$ and $N$ for the two special cases of perfectly conducting and insulating inclusions. The effective conductivity of the composite is found to approach that of the continuous matrix in higher dimensions.
\end{abstract}

Key words. Laplace equation in $N$-dimensions, random walk, effective conductivity or diffusivity in inhomogeneous media

AMS(MOS) subject classification. 35J05

1. Introduction. One of the classical problems in physics is the determination of the effective or macroscopic property of a two-phase medium consisting of inclusions of one material of known shape embedded homogeneously into a continuous matrix of another having physical properties different from its own. When the inclusions are spheres and their amount is small, the effective thermal conductivity $k^{*}$ of the composite medium is given by the well-known Maxwell relation

$$
\frac{k^{*}}{k}=1+\frac{3(\alpha-1)}{\alpha+2} \phi+O\left(\phi^{2}\right)
$$

where $k$ is the thermal conductivity of the continuous matrix phase, $\alpha$ is the ratio of thermal conductivities of the inclusions and the matrix, and $\phi$ is the volume fraction of the spheres. The above result is valid for small $\phi$ and applies to arbitrary size and spatial distribution of the spherical inclusions. For the cases where either $\phi$ is not small or where $\alpha$ differs greatly from unity, it is necessary to specify the size and spatial distributions of the inclusions to obtain more accurate estimates of $k^{*}$. Determination of $k^{*}$ when these distributions are given has been the subject of numerous articles in the past. For example, $k^{*}$ for the complete range of $\phi$ for the equal size spherical or cylindrical inclusions in both the periodic and the random spatial distributions have been obtained [4]-[6], [8], [9]. It is found that unless $\alpha \gg 1$ and $\phi$ approaches its maximum allowable value (corresponding to the case when the inclusions begin to touch their neighbors) the estimates obtained for the periodic arrangements of the inclusions compare well with those obtained for the random arrangements.

The effective conductivity approaches infinity at the maximum volume fraction of the perfectly conducting inclusions $(\alpha=\infty)$ either as the inverse square root of the minimum gap between the inclusions in case of the cylindrical (two-dimensional) inclusions or logarithmically in case of the spherical (three-dimensional case) inclusions, and thus the exact arrangement of the particles becomes an important

* Received by the editors July 20, 1988; accepted for publication February 9, 1989.

† Department of Mechanical Engineering, The Johns Hopkins University, Baltimore, Maryland 21218. Permanent address, Department of Chemical Engineering and Material Science, Syracuse University, Syracuse, New York 13244. This work was supported by National Science Foundation grant CBT-8800451. 
variable in determining $k^{*}$ in the limiting case of $\alpha \rightarrow \infty$ and $\phi \rightarrow \phi_{t}$, the maximum allowable volume fraction.

Brady [1] has considered a problem of determining the well-known $O(\phi)$ Einstein correction to the effective viscosity of the suspension of neutrally buoyant $N$ dimensional hyperspheres (or simply the $N$-dimensional "spheres") for arbitrary $N$ $(N \geqq 2)$. The problems of determining $k^{*}$ or the effective viscosity for the general case of $\mathrm{N}$-dimensional "spherical" particles serve as interesting home assignments to the graduate students taking their first course in fluid mechanics and heat transfer. As shown below, the coefficient of $\phi$ in (1) in this general case becomes $N(\alpha-1) /(\alpha+N-$ $1)$, which increases linearly with $N$ when $\alpha=\infty$. The students fascinated by such exercises question what would happen at large $\phi$. Although the coefficient of $\phi$ increases linearly with $N$, the effective conductivity is expected to become less sensitive to the geometrical arrangement of the "spheres" even when $\alpha=\infty$ and as the "spheres" begin to touch their neighbors for $N \geqq 4$, in accordance with the trend set by $N=2$ and 3 . The purpose of this note is to give the estimates of $k^{*}$ for arbitrary $N$ and $\phi$ for the case of simple periodic arrangement. Although we are not aware of any physical situation where the results for the general $N$-dimensional case may be directly applicable, such an effort, i.e., the determination of $k^{*}$ for an arbitrary $N$, is undertaken largely due to the general interest in the problem of determining the macroscopic properties of the inhomogeneous media. Since the boundary value problem to be solved appears in many other physical situations, including the electrical conduction and the diffusion or the "random-walk" problems, the solution presented here may perhaps have utility in some situations other than the case of thermal conduction considered here.

2. Formulation and the method of solution. The boundary value problem to be solved is the familiar Laplace equation for the conduction of heat subject to the continuity of the temperature and flux at the boundaries of the $N$-dimensional "spheres" of radii $a$ with their centers coinciding with an $N$-dimensional lattice. Since the effective conductivity is expected to remain bounded for $N \geqq 4$, it would suffice to calculate the first few terms in the asymptotic expansion of $k^{*}$ for small $\phi$. The leading order effect of the particles at $\phi \rightarrow 0$ can be approximated as that due to the point thermal dipoles. Since the temperature field due to a point dipole decays only as $r^{1-N}$ at large $r$, where $r$ is the distance from the dipole, it can be shown that the sum total due to the presence of many dipoles present in the medium is not absolutely convergent. The ways of overcoming the difficulties of this kind are now well known and here we follow Zuzovski and Brenner [10] and Sangani and Acrivos [7] by beginning with the singular function $S$ for the Laplace equation. This function satisfies the differential equation

$$
\nabla^{2} S(r)=\lambda_{N}\left[\frac{1}{\tau}-\sum_{\mathbf{r}^{\alpha}} \delta\left(\mathbf{r}-\mathbf{r}^{\alpha}\right)\right]
$$

where $\mathbf{r}^{\alpha}$ are the lattice points, $\tau$ is the "volume" of the unit cell, and $\lambda_{N}$ is a constant to be specified later. The sink term $1 / \tau$ is needed in the definition of $S$ to satisfy the overall heat balance for $S$. It is evident that any derivative of $S$ will satisfy the Laplace equation so that the temperature at any point outside the "spheres" can be expressed as

$$
T=\mathbf{G}(\cdot) \mathbf{x}+\sum_{p=1}^{\infty} \mathbf{G}(\cdot) \mathbf{A}_{p+1}(\cdot)^{p} \nabla_{(p)} S
$$


where $\mathbf{G}$ is the imposed temperature gradient, $\mathbf{x}$ is the position vector, $\mathbf{A}_{p+1}$ is a tensor of rank $p+1, \nabla_{(p)}$ is the gradient operator of rank $p$, and $(\cdot)^{p}$ implies a $p$-fold inner product. Thus, for example, in the Einstein notation

$$
\mathbf{G}(\cdot) \mathbf{A}_{p+1}(\cdot)^{p} \nabla_{(p)}=G_{i_{1}} A_{i_{1} i_{2} \ldots i_{p+1}} \frac{\partial^{p}}{\partial x_{i_{2}} \partial x_{i_{3}} \cdots \partial x_{i_{p+1}}} .
$$

The tensors $A$ depend only on the geometry of the array. Since any derivative of $S$ satisfies the Laplace equation, we will require that the trace of $\mathbf{A}$ with respect to any two of the indices $i_{2}, i_{3}, \cdots, i_{p+1}$ must be zero. To determine $\mathbf{A}$ from the boundary conditions that the temperature and flux are continuous everywhere, and in particular at the surface of the "spheres," we express the temperature inside the particle situated at the origin in terms of the growing harmonics and in terms of both the growing and decaying harmonics outside the "sphere." The relationship between the coefficients of the growing and decaying harmonics is then determined by satisfying the aforementioned boundary conditions at the surface of the "sphere" situated at the origin. Thus, let us assume, without loss of generality, that $G_{i}=\delta_{i 1}$ and that near $r=0$

$$
S=g+g^{\prime}
$$

where $g$ is the singular part of $S$. Also let us choose $\lambda_{N}$ in (2) such that the singular part is given by

$$
g= \begin{cases}r^{2-N}, & N \geqq 3 \\ \log (1 / r), & N=2 .\end{cases}
$$

The temperature near $r=0$ can be expressed now in terms of the derivatives of $g$ as

$$
T=\left\{\begin{array}{l}
\sum_{p} \mathbf{f}_{p}(\cdot)^{p} \nabla_{(p)} g+r^{2 p+N-2} \mathbf{h}_{p}(\cdot)^{p} \nabla_{(p)} g, \quad r \geqq a, \\
\sum_{p} r^{2 p+N-2} \mathbf{h}_{p}^{\prime}(\cdot)^{p} \nabla_{(p)} g, \quad r \leqq a
\end{array}\right.
$$

where $\mathbf{f}, \mathbf{h}$, and $\mathbf{h}^{\prime}$ are the coefficients of the decaying and growing harmonics. On satisfying the boundary conditions at $r=a$, we obtain

$$
\mathbf{f}_{p}=-L_{p} \mathbf{h}_{p} a^{2 p+N-2}
$$

with

$$
L_{p}=\frac{\alpha-1}{\alpha+1+(N-2) / p} .
$$

On expanding $T^{\prime}$, the regular part of the temperature, near $r=0$ and expressing each term in the expansion in terms of growing harmonics, it can be shown that

$$
h_{p}=\frac{(-1)^{p}}{p !} \frac{(N-4) ! !}{[N+2(p-2)] ! !} \nabla_{(p)} T^{\prime}(0), \quad p \geqq 1
$$

with $(-2) ! ! \equiv(-1) ! ! \equiv(0) ! !=1$. In deriving $(10)$, we have used the fact that $\nabla^{2} T^{\prime}=0$. Now, a comparison of (7) and (3) suggests that

$$
\mathbf{f}_{p}=\mathbf{e}_{1}(\cdot) A_{p+1}
$$

where $\mathbf{e}_{1}$ is the unit vector in the $x_{1}$-direction that is chosen to coincide with the direction of the mean temperature gradient in the composite medium. The regular part $T^{\prime}$ can be obtained by substituting $g^{\prime}$ for $S$ in (3) so that on using (10) we have

$$
\mathbf{f}_{p}=\tilde{L}_{p} a^{2 p+N-2} \nabla_{(p)}\left\{x_{1}+\sum_{q=1}^{\infty} \mathbf{f}_{q}(\cdot)^{q} \nabla_{(q)} g^{\prime}\right\}_{r=0}
$$


where

$$
\tilde{L}_{p}=\frac{-(-1)^{p}}{p !} \frac{(N-4) ! !}{[N+2(p-2)] ! !} L_{p} .
$$

Now (12) can be solved by successive approximation when $a$ is small, i.e., when the dispersed phase "volume" fraction $\phi$ is small. We will solve (12) for the special case of a simple "cubic" lattice for which the $N$ basis vectors are along the $N$-coordinate axes. For this lattice, the symmetry conditions require that $f_{(2 p)} \equiv 0$ and the explicit expressions for the first few f's are obtained as follows:

$$
\begin{gathered}
\mathbf{f}_{7}=F a^{N+12} \tilde{L}_{7}\left[\nabla_{(7) 1} g^{\prime}(0)+O\left(a^{N+4}\right)\right], \\
\mathbf{f}_{5}=F a^{N+8} \tilde{L}_{5}\left[\nabla_{(5) 1} g^{\prime}(0)+a^{N+4} \tilde{L}_{3} \nabla_{(3) 1} g^{\prime}(0)(\cdot)^{3} \nabla_{(8)} g^{\prime}(0)+\left(a^{N+8}\right)\right], \\
\mathbf{f}_{3}=F a^{N+4} \tilde{L}_{3}\left[\nabla_{(3) 1} g^{\prime}(0)+a^{N+4} \tilde{L}_{3} \nabla_{(3) 1} g^{\prime}(\cdot)^{3} \nabla_{(6)} g^{\prime}\right. \\
\left.+a^{N+8} \tilde{L}_{5} \nabla_{(5) 1} g^{\prime}(0)(\cdot)^{5} \nabla_{(8)} g^{\prime}+O\left(a^{N+12}\right)\right], \\
\mathbf{f}_{1}=F \delta_{i 1} \quad a^{N} \tilde{L}_{1}\left[1+F\left\{\nabla_{1,1} g^{\prime}(0)+a^{N+4} \tilde{L}_{3}\left(a^{N+4} \tilde{L}_{3} \nabla_{(3) 1} g^{\prime}(\cdot)^{3} \nabla_{(6)} g^{\prime}(0)+\nabla_{(3) 1} g^{\prime}(0)\right)\right.\right. \\
\left.\left.\cdot(\cdot)^{3} \nabla_{(3) 1} g^{\prime}(0)+a^{N+8} \tilde{L}_{5} \nabla_{(5) 1} g^{\prime}(\cdot)^{5} \nabla_{(5) 1} g^{\prime}(0)+O\left(a^{N+12}\right)\right\}\right] \delta_{11}
\end{gathered}
$$

where $F$ represents the magnitude of the thermal dipole in the $x_{1}$-direction. It can be shown that the effective conductivity of the composite medium is related to $F$ by

$$
\frac{k^{*}}{k}=1+\phi C_{N} F a^{-N}
$$

with

$$
C_{N}= \begin{cases}N(N-2), & N \geqq 3, \\ N, & N=2 .\end{cases}
$$

In (15), $\phi$ is the "volume" fraction of the "spheres" and is defined by

$$
\phi=\frac{V_{N}^{\prime} a^{N}}{\tau}
$$

where $V_{N}^{\prime}$ is the "volume" of the "sphere" of unit radius and $\tau=h^{N}$ is the "volume" of the unit cell with each side of the unit cell being $h$ units. It can be shown that $V^{\prime}$ satisfies the following recursion relation:

$$
V_{N}^{\prime}=\frac{2 \pi}{N} V_{N-2}^{\prime}
$$

with

$$
V_{2}^{\prime}=\pi \quad \text { and } \quad V_{3}^{\prime}=\frac{4 \pi}{3} .
$$

Or, alternatively, combining (18) and (19), we have

$$
V_{2 N}^{\prime}=\frac{(2 \pi)^{N}}{(2 N) ! !}, \quad V_{2 N+1}^{\prime}=\frac{2(2 \pi)^{N}}{(2 N+1) ! !} .
$$


Finally, to determine $\lambda_{N}$ in (2) so that the singular part of $S$ is given exactly by (6), we must apply the divergence theorem to a small "sphere" of radius $\varepsilon$ centered around $r=0$ and take the limit $\varepsilon \rightarrow 0$. This yields

$$
\lambda_{N}= \begin{cases}N(N-2) V_{N}^{\prime}, & N \geqq 3, \\ N V_{N}^{\prime}, & N=2,\end{cases}
$$

or simply, $\lambda_{N}=V_{N}^{\prime} \cdot C_{N}$.

3. Results. To determine the effective conductivity, we need to substitute $F$ given by (14d) into (15) and evaluate the various derivatives of $g^{\prime}$ appearing in (14d). These derivatives depend on $h$, the size of the unit cell. The final result can be expressed in terms of a nondimensional radius $\chi$ defined by

$$
\chi=\frac{2 a}{h}=\left(\frac{\phi}{\phi_{t}}\right)^{1 / N}
$$

where $\phi_{t}$ is the maximum allowable "volume" fraction of the "spheres" packed in the simple "cubic" array and corresponds to the case where each "sphere" in the array just begins to touch its neighboring "spheres." $\phi_{t}$ is related to $V_{N}^{\prime}$ by

$$
\phi_{t}=V_{N}^{\prime} 2^{-N}
$$

and has the numerical values $0.785,0.524,0.308,0.1645,0.0807$, and 0.0369 for $N=2$, $3,4,5,6$, and 7 , respectively.

In evaluating the constants appearing in (14d), we may chose $a=\chi$ and $h=2$ with no loss of generality. The result of combining (14d) and (15) can now be expressed as

$$
\frac{k^{*}}{k}=1+\frac{\phi_{t} \chi^{N} N L_{1}}{\bar{F}}
$$

with

$$
\bar{F}=1-\tilde{L}_{1}\left[e_{1} \chi^{N}+\frac{\tilde{L}_{3} e_{2} \chi^{2 N+4}}{1-e_{3} \tilde{L}_{3} \chi^{N+4}}+\tilde{L}_{5} e_{4} \chi^{2 N+8}+O\left(\chi^{2 N+12}\right)\right]
$$

where the constants $e_{1}-e_{4}$ are related to various derivatives of $g^{\prime}$ evaluated at $r=0$. It may be noted that, to $O\left(\chi^{N}\right)$, the expression (24) reduces to the Maxwell result (1) with the coefficient of $\phi$ given by

$$
N L_{1}=\frac{(\alpha-1) N}{\alpha+N-1}
$$

in accordance with the well-known results for $N=2$ and $N=3$. The explicit expressions for the constants $e_{1}-e_{4}$ are:

$$
\begin{aligned}
& e_{1}=\frac{\partial^{2} g^{\prime}}{\partial x_{1}^{2}}(0), \quad e_{2}=\left[\nabla_{(3) 1} g^{\prime}(\cdot)^{3} \nabla_{(3) 1} g^{\prime}\right]_{r=0}, \\
& e_{2} e_{3}=\left[\nabla_{(3) 1} g^{\prime}(\cdot)^{3} \nabla_{(6)} g^{\prime}(\cdot)^{3} \nabla_{(3) 1} g^{\prime}\right]_{r=0}, \\
& e_{4}=\left[\nabla_{(5) 1} g^{\prime}(\cdot)^{5} \nabla_{(5) 1} g^{\prime}\right]_{r=0} .
\end{aligned}
$$

To evaluate the derivatives of $g^{\prime}$, we note first of all that an integral representation of $S$ satisfying (2) is

$$
S=\sum_{\alpha}\left\{g\left(\mathbf{r}-\mathbf{r}^{\alpha}\right)-\frac{\lambda_{N}}{\tau} \int_{\tau^{\alpha}} g\left(\mathbf{r}-\mathbf{r}^{\prime}\right) d \mathbf{r}^{\prime}\right\}
$$


so that near $r=0$

$$
g^{\prime}=\sum_{r^{\alpha} \neq 0} g\left(r-r^{\alpha}\right)-\frac{\lambda_{N}}{\tau} \sum_{\alpha} \int_{\tau^{\alpha}} g\left(r-r^{\alpha}\right) d r^{\prime} .
$$

Here $\tau^{\alpha}$ is the "volume" occupied by the unit cell labeled " $\alpha$." It may be noted that although the sums in (29) do not converge individually, the difference between the two sums converges so that $g^{\prime}$ is finite. To evaluate the derivatives of $g^{\prime}$, however, we may ignore the second sum on the right-hand side of (29) provided that the sum of the derivatives of the terms in the first sum is absolutely convergent. Since $g \sim r^{2-N}$, this means that the derivatives of order greater than or equal to three can be computed directly by differentiating the first term in (29). Thus

$$
\nabla_{(p)} g^{\prime}(0)=\sum_{\alpha \neq 0} \nabla_{(p)} g\left(0-r^{\alpha}\right), \quad p \geqq 3 .
$$

To evaluate the second derivative of $g^{\prime}$ at $r=0$ needed in determining $e_{1}$ via (27), we use a different method. Here, because of the "cubic" symmetry of $g^{\prime}$ and, because of (2), we observe that $g^{\prime}$ near $r=0$ must be of the form

$$
g^{\prime}=\text { const. }+\frac{\lambda_{N} r^{2}}{2 N \tau}+O\left(r^{4}\right)
$$

The second-order derivative at $r=0$ is now readily evaluated, yielding

$$
e_{1}=\frac{\lambda_{N}}{N \tau}
$$

Taking $h=2$, the above expression gives $e_{1}=\pi / 4$ and $\pi / 6$, respectively, for $N=2$ and 3, in agreement with the results obtained by Perrins, McKenzie, and McPhedran [6] and Zuzovski and Brenner [10]. Next, to evaluate $e_{2}$, let us define

$$
\Lambda_{(4)}=\Lambda_{i j k l}=\left[\frac{\partial^{4} g^{\prime}}{\partial x_{i} \partial x_{j} \partial x_{k} \partial x_{l}}\right]_{r=0} \text {. }
$$

Since $\nabla^{2} g^{\prime}=0$, we note that the trace of $\Lambda$ with respect to any two of its indices must be zero. Also, $\Lambda$ must be symmetric with respect to all of its indices and invariant under $90^{\circ}$ rotation of the coordinate axes since the unit cell is "cubic." A most general expression for $\Lambda_{(4)}$ satisfying all of the above conditions involves only one scalar:

$$
\Lambda_{i j k l}=\lambda_{1} \hat{\delta}_{i j k l}=\lambda_{1}\left\{\delta_{i j k l}-I_{i j k l}\right\}
$$

with

$$
I_{i j k l}=\frac{1}{(N+2)}\left[\delta_{i j} \delta_{k l}+\delta_{i l} \delta_{j k}+\delta_{i k} \delta_{j l}\right]
$$

and $\delta_{(4)}=\delta_{i j k l}$ equals unity if $i=j=k=l$, and zero otherwise. It can be shown now that

$$
e_{2}=\Lambda_{(3) 1}(\cdot)^{3} \Lambda_{(3) 1}=\frac{1}{N} \Lambda_{(4)}(\cdot)^{4} \Lambda_{(4)}=\lambda_{1}^{2} \frac{(N-1)}{N+2} .
$$

The scalar $\lambda_{1}$ can be evaluated by taking $i=j=k=l=1$, whence we obtain

$$
\lambda_{1}=\frac{N+2}{N-1}\left[\frac{\partial^{4} g^{\prime}}{\partial x_{1}^{4}}\right]_{r=0} .
$$


Substituting (30) into (37) and using either a method of theta functions outlined in Born and Misra [2] or by a direct summation method using a computer, $\lambda_{1}$ can be determined for various $N$. Similarly, the constant $e_{4}$ is evaluated from

$$
e_{4}=\frac{1}{N} \Lambda_{(6)}(\cdot)^{6} \Lambda_{(6)}
$$

where $\Lambda_{(6)}$ must be of the form

$$
\Lambda_{(6)}=\lambda_{2}\left\{\delta_{6}-\frac{1}{3(N+4)} I_{2} I_{4}-\frac{1}{N+8} I_{2} \hat{\delta}_{4}\right\}=\lambda_{2} \hat{\delta}_{6}
$$

After some lengthy algebra, it can be shown that

$$
\hat{\delta}_{6}(\cdot)^{6} \hat{\delta}_{6}=\frac{N(N-1)(N-2)}{(N+4)(N+8)}
$$

and

$$
\lambda_{2}=\frac{(N+4)(N+8)}{(N-1)(N-2)}\left[\frac{\partial^{6} g^{\prime}}{\partial x_{1}^{6}}\right]_{r=0} .
$$

The constant $e_{3}$ can also be shown to be related to $\lambda_{2}$ by

$$
e_{3}=\lambda_{2} \frac{(N-2)(N+2)}{(N+4)(N+8)} \text {. }
$$

Finally, to evaluate the derivatives required by expressions (37) and (41), we may make use of the following general result:

$$
\frac{\partial^{p}}{\partial x_{1}^{p}} r^{2-N}=\sum_{s=0}^{p / 2}(-1)^{p-s} \frac{(N+2 p+2 s-4) ! !}{2^{s} s !(N-4) ! !} \frac{p !}{(p-2 s) !} x_{1}^{p-2 s} r^{2-N-2 p+2 s} .
$$

These derivatives were evaluated with the help of a computer program and the computed values of $e_{1}-e_{4}$ are given in Table 1 for various $N$. The resulting expression for $N=3$ is in perfect agreement with that reported by Sangani and Acrivos [7] to the level of approximation, i.e., $O\left(\chi^{4 N+8}\right)$, considered in the present study. Although these authors employed a technique similar in principle to the one employed here, the resulting algebra was quite different as they used expansions in the Legendre functions instead of the tensor calculus employed here. The perfect agreement between the two is gratifying in view of the rather tedious algebra involved in both techniques. The numerical values of $k^{*} / k$ as a function of $\chi$ and $N$ for $\alpha=\infty$ and $\alpha=0$ are shown in Figs. 1 and 2, where the exact results for $N=2$ and 3 as given by Perrins, McKenzie,

TABLE 1

The constants $e_{1}-e_{4}$ and $\phi_{t}$ for various $N$.

\begin{tabular}{cccccl}
\hline$N$ & $e_{1}$ & $e_{2}$ & $e_{3}$ & $e_{4}$ & $\phi_{t}$ \\
\hline 3 & 0.5236 & 13.586 & 8.062 & 400.72 & 0.5236 \\
4 & 0.6169 & 66.761 & 37.195 & 5533.9 & 0.308 \\
5 & 0.4935 & 128.02 & 82.961 & 21911.6 & 0.1645 \\
6 & 0.3230 & 153.31 & 131.48 & 47270 & 0.0807 \\
7 & 0.1846 & 136.94 & 169.29 & 70056 & 0.0369 \\
\hline
\end{tabular}




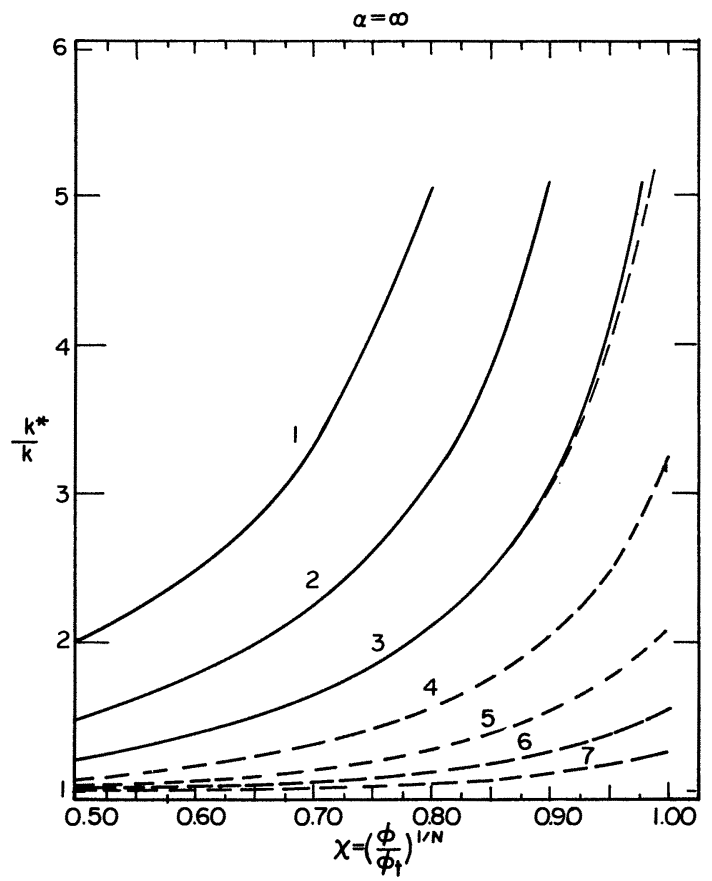

FIG. $1 . k^{*} / k$ as a function of $\chi$ and $N$ for perfectly conducting $(\alpha=\infty)$ inclusions. The solid lines for $N=1,2$, and 3 correspond to the exact results, whereas the dotted lines for $N=3$ to 7 correspond to the asymptotic expression derived in the present study.

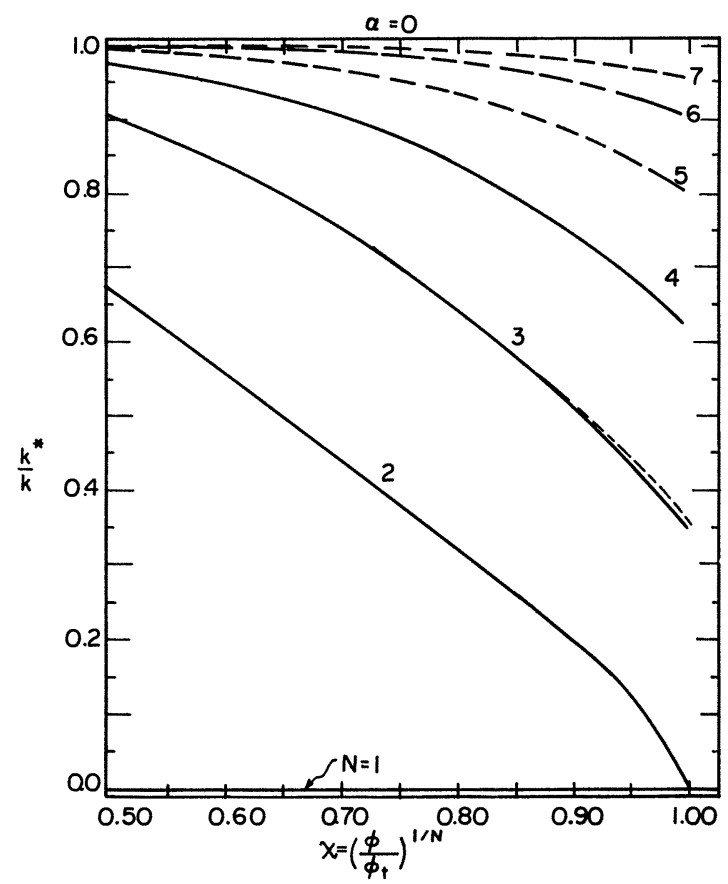

FIG. 2. $k^{*} / k$ as a function of $\chi$ and $N$ for perfectly insulating $(\alpha=0)$ inclusions. The solid lines for $N=1$ to 3 correspond to the exact results whereas the dotted lines for $N=3$ to 7 correspond to the asymptotic expression derived in the present study. 
and McPhedran [6] and McPhedran and McKenzie [4] are shown along with the asymptotic results for $N=3,4,5,6$, and 7 as calculated from (24), (25), and Table 1 . The exact result for $N=1$ is trivially obtained from

$$
\frac{k^{*}}{k}=\left(\frac{\chi}{\alpha}+\frac{1-\chi}{1}\right)^{-1},
$$

which corresponds to the effective conductivity of a composite consisting of parallel slabs. It may also be noted that $k^{*} / k$ approaches infinity as $\chi \rightarrow 1$ for $\alpha=\infty$ and $N=2$ and 3 as [6], [7]

$$
\frac{k^{*}}{k}= \begin{cases}-\frac{\pi}{2} \log (1-\chi)-0.69 & (N=3, \alpha=\infty), \\ \frac{\pi}{(2(1-\chi))^{1 / 2}}-2.0 & (N=2, \alpha=\infty) .\end{cases}
$$

This divergent behavior of $k^{*}$ for $N=2$ or 3 at $\chi=1$ and $\alpha=\infty$ cannot be captured, in general, with only few terms in the expansion near $\chi=0$. However, we find that the asymptotic expansion given by (24) and (25) for $N=3$ gives surprisingly accurate estimates of $k^{*}$ up to $\chi=0.95$ even for $\alpha=\infty$. For larger $N$, we expect (24) and (25) to give fairly accurate estimates even at $\chi=1$. These estimates for $k^{*} / k$ at $\chi=1$ and $\alpha=\infty$ are $3.23,2.09,1.55$, and 1.27 , respectively, for $N=4,5,6$, and 7 . Thus, we see that the effective conductivity of the composite approaches that of the matrix in higher dimensions even though the coefficient of $O(\phi)$ in the expression for $k^{*} / k$ has been found to increase linearly with $N$. This is because the maximum allowable "volume" fraction in higher dimensions decreases very rapidly, in fact, exponentially with $N$.

For $\alpha=0, k^{*} / k$ approaches zero as $\chi \rightarrow 1$ for $N=2$ while it approaches a constant for $N \geqq 3$. (Note that $k^{*} \equiv 0$ at all $\chi$ for $N=1$.) As shown by Keller [3]

$$
k^{*}(\alpha=\infty)=k^{2} / k^{*}(\alpha=0)
$$

for $N=2$. At $\chi=1$, the "spheres" (or circles) in $N=2$ provide infinite resistance to the heat flux and hence $k^{*} \rightarrow 0$ as $\chi \rightarrow 1$. For $N>2$, however, the flux through the matrix remains finite even at $\chi=1$ and $\alpha=0$, so that $k^{*} / k$ approaches unity very rapidly in higher dimensions.

\section{REFERENCES}

[1] J. F. BRADY, Einstein viscosity correction in N-dimensions, Internat. J. Multiphase Flow, 10 (1984), pp. 113-114.

[2] M. Born AND R. D. Misra, On the stability of crystal lattices IV, Proc. Camb. Phil. Soc., 36 (1940), pp. 466-478.

[3] J. B. KELlER, Conductivity of a medium containing a dense array of perfectly conducting spheres or cylinders of nonconducting cylinders, J. Appl. Phys., 34 (1963), pp. 991-993.

[4] R. C. MCPhedran And D. R. MCKenzIE, Conductivity of lattices of spheres I. The simple cubic lattice, Proc. Roy. Soc. London, A359 (1978), pp. 45-62.

[5] D. R. McKenzie, R. C. MCPhedran, AND G. M. Derrick, Conductivity of lattices of spheres II. The body-centered and face-centered cubic lattices, Proc. Roy. Soc. London, A362 (1978), pp. 211-232.

[6] W. T. PERrins, D. R. MCKEnZIE, ANd R. C. MCPhEdran, Transport properties of regular arrays of cylinders, Proc. Roy. Soc. London, A369 (1979), pp. 207-225.

[7] A. S. SAngAni And A. Acrivos, Effective conductivity of a periodic array of spheres, Proc. Roy. Soc. London, A386 (1983), pp. 263-275. 
[8] A. S. SANGANI AND C. YAO, Bulk thermal conductivity of composites with spherical inclusions, J. Appl. Phys., 63 (1988), pp. 1334-1341.

[9] - Transport processes in random arrays of cylinders I. The thermal conduction, Phys. Fluids, 31 (1988), pp. 2426-2434.

[10] M. ZuZOVSKI AND H. BRENNER, Effective conductivities of composite material's composed of cubic arrangements of spherical particles embedded in an isotropic matrix, J. Appl. Math. Phys. (Z. Angew. Math. Phys.), 28 (1977), pp. 979-992. 